\title{
JOURNAL
}

OF

\section{THE ROYAL SANITARY INSTITUTE}

\section{MEDICAL INSPECTION : \\ ITS RELATIONSHIP TO THE HOME}

LIFE OF THF CHILDREN.

By RALPH P. WILLIAMS, M.D.,

Professor of Public Health in the University of Sheffield. (MEMBER.)

Read at Sessional Meeting, Sheffield, March 12th, 1909.

A RECENT Circular of the Board of Education, which lays down the A lines on which the annual report of the school medical officer is to be written, states that information is to be given as to the physique of the children, in relation to the social and industrial conditions under which they live.

At the present stage of medical inspection this will not be an easy part of the report to write, although some valuable work has been done on the subject.

I will briefly refer to some aspects of the home life of the children attending the schools in poor districts, these being the children whom systematic medical inspection will particularly benefit, in removing, as far as possible, an environment which cannot be other than harmful to the proper development of the young child.

The first condition which strikes the medical inspector in examining the children in a poor district school is the large number who are verminous. This condition may affect either head or clothing, or both. This is apparently the most easily prevented of all preventable diseases, but in practice the question is not so simple.

The teacher is our first helper in remedying the condition, and by constant attention may achieve a great deal, aided by suitable cards to be sent home to parents (these cards containing instructions as to cleansing).

voL. $\mathbf{x x x}$. No. 4. 
The time of the teacher is, however, fully occupied, and it is quite impossible for her to obtain anything approaching complete success.

The school nurse is indispensable, and at present this work of obtaining treatment for such conditions is a large part of her duties. At the present time in Sheffield three Queen's Nurses are engaged in this work.

The method we are adopting is to restrict the work of the school nurses to certain poor-district schools. The whole of the children in attendance are examined with regard to cleanliness, a list being made of the dirty ones. Cards are sent to the less severe cases, and home visits paid to those whose condition warrants this. A second visit is paid to the same school in about three weeks, the dirty children are then re-examined. Cards are sent as before, or home visits made, and the mothers again instructed as to cleansing where necessary.

On the third visit the school medical officer and the chief lady sanitary inspector visit the school with the nurse. The dirty children are again examined, and where necessary I visit the homes, and either warn the parents, or, if the condition of the children and the home is very bad, I apply for a warrant (under section 10 of the Prevention of Cruelty to Children Act, 1904) to remove the children to a place of safety, the cottage homes of the Poor Law Union.

In one school, on September 1st, 194 girls were examined by the nurse; of these, 4 were found to be clean, 102 had nits only, 33 head lice and nits, and 55 body lice and nits. As a result of the nurse's visits and instructions, in two months' time the number of clean children increased from 4 to 43 , whilst the verminous children numbered 105,10 , and 16 respectively.

On examining the same school after the Christmas holidays, the conditions were found to have partially recurred; the figures then being 4 clean (girls with short hair), 138 nits only, 13 head lice and nits, 37 body lice and nits. The verminous children were, however, in a much more cleanly state than they were at the beginning of the previous term.

This indicated that the furniture and the bedding of the home was infected, and that many. parents gave up active measures to keep the children clean, whilst they were away from the strict supervision of the teacher and the nurse.

The staff of women sanitary inspectors is of the greatest value in this work; they are called in at once by the school nurse if the home is found to be in a very dirty condition, and by their instructions many homes are cleansed. It was found necessary, however, to serve notices for cleansing (under Section 91 of the Public Health Act) in 70 cases arising out of 1,398 school complaints during the past year in Sheffield. 
Before leaving this subject I will quote one case of rather more than usual interest.

Three boys aged 13,9 , and 5 years respectively were found by me attending school in a very neglected and verminous state; they were reported to the women sanitary inspectors, the home visited, and the parents warned. They removed to another district, and were lost sight of for about three months.

I then found the children in a similar condition in another school, and ascertained that they were living with their father in a house let in lodgings, the reputation of which was well known to the police.

This house I visited with Dr. Scurfield shortly after midnight. The three boys were asleep in the same bed as the father. They were naked with the exception of a sheet, and it was found that the air space of the room was considerably less than that required by the local by-laws.

In the morning, therefore, action was taken, the children removed to the cottage homes, and the father prosecuted for neglect. He was remanded in order that he might obtain employment and thus establish a home for his children. This he failed to do, and as it was proved by the police that he had had ample opportunity to obtain work, he was sentenced to four months hard labour. The lodging house keeper was, on conviction, fined two pounds in respect of the overcrowding.

Cases of neglect may be dealt with legally under two Acts : (1) Prevention of Cruelty to Children Act, 1904, section 10 (quoted above); (2) Education Act.-Under this Act a High Court decision has been obtained in which it is stated that "an attendance is not an attendance in law unless the child is in a fit condition to attend school."

If the child comes to school in a verminous state it can then be excluded and the parents prosecuted for the non-attendance of the child.

This latter course we have not adopted as the result is usually that the father is fined 10s. The fining of poor parents is not likely to benefit the home or the child, and so we have felt that the former Act is the better, the worst cases being selected, and in the five cases we have prosecuted since last July sentences have been imposed varying from six to one month's imprisonment. Every chance is given to the parents to improve, and in most of the prosecutions the solicitor is instructed by us to ask for a remand for one to three months, in order that the parents may have every opportunity of mending their ways.

The Children Act (1908) which comes into force on April 1st next, will considerably strengthen the school medical officers' hands. Under section 12, parents or guardians who ref use to obtain treatment for children 
with defective eyesight or hearing are liable to penalties, and under section 122 (which deals with verminous children) the school medical officer may remove the child to suitable premises and have it cleansed without previously obtaining any warrant, if a notice to cleanse the child within twenty-four hours has not been complied with.

In the cleansing of verminous children the poor labour under the greatest difficulties, especially those who are unfortunate enough to have reached the level of living in what are known in Sheffield as furnished rooms, that is, houses let in lodgings.

Indeed, in my opinion, the mothers who keep their children clean deserve the highest praise, as it is difficult to realise the physical strain on one woman of doing the house-cleaning, washing and cooking, besides the work entailed in dressing and bathing of perhaps half-a-dozen children : this may have to be done in a house where the water has to be fetched from a standpipe and heated for bathing purposes in kettles or pans.

I do think we shall not be removing much of this parents' responsibility if we establish school baths, and let the children be washed there weekly, instead of in the copper or "set-pot," as I understand is commonly practised in northern cities.

With regard to pulmonary tuberculosis, Sheffield is fortunate in this disease being compulsorily notifiable, and I have arranged that the names of those children who are living in the same house as a notified case should be sent to the School Medical Officer's Department every week. In this way particular attention will be paid during the examination of these children's chests as to the possible presence of early tuberculous disease of the lungs. It is to be hoped that in the immediate future, crowded areas will follow the example of London and Bradford in establishing open-air schools for the treatment of children with anæmia, early phthisis, and other debilitating diseases. The Sheffield Education Committee are at present considering the question of opening one of these schools.

There is no doubt of the defective nutrition of a large number of our school children. For this condition improper feeding and underfeeding is largely responsible. The latter in times of stress and unemployment is no doubt an important factor in many cities. At the present time about 3,700 breakfasts and 1,800 dinners are being given daily in Sheffield to needy children.

Improper feeding is, without doubt, the principal factor in defective nutrition when plenty of work is available. I have seen strong tea being siven to young children; and lately one child brought a large crust of 
bread soaked in the vinegar of pickled cabbage to serve as the midday meal. The provision of a suitable midday meal, for which all should pay who are able, would be one solution to a difficult problem.

The teaching of cookery and housewifery in our girls' schools should be greatly extended, the time which is being given to it at present being inadequate. Girls over 11 years of age receive a 48-hour course of instruction in cookery during the educational year.

The question of work done out of school hours is one which will come before the notice of the school medical officer. In Sheffield the number of children who are half-timers is not great, 467 out of 78,866 on the rolls. More than half these half-timers are girls engaged in domestic work at home, and one third are over 13 years of age.

In examining the older boys and girls it is important to ascertain if they are interfering with their educational work by outside occupation, whether they be half or whole-timers at school. The following are instances:-

1. Boy, aged 12 years, in Standard VII., half-timer. School-day work: 9 to 12 noon, lathers for barber; 2 to 4.30 p.m., school work; 5 to 8 p.m., lathers for barber. Saturday: 9 a.m. to 10 p.m., lathers for barber. Earns 3s. 6d. a week.

2. Boy aged 12 years, in Standard VII., whole-timer. School-day work: 6 to 8 a.m., newspapers; 9 to 12 , school; 12.30 to 1.30 , grocer's shop; 2 to 4.30 , school ; 7 to 8.15 p.m., grocer's shop. Saturday : 6 to 8

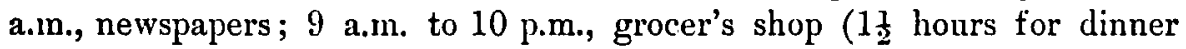
and tea). Earns 4 s. a week.

In both these cases the local by-laws had been broken. Enquiries have been made through the women sanitary inspectors, and the hours during which the children are working have been reduced.

In both cases the boys appeared bright, and their position in the school was good. This is the very kind of boy who becomes a worker out of school hours, but there is no doubt, medically speaking, that these children are doing thernselves physical harm by such work, and from the educational point of view that they cannot benefit by instruction in the same way as if they approached their work fresh from sleep or play.

In a boy who is worked in this way, home lessons are, of course, entirely out of the question. This equally applies to the great mass of children in poor district homes.

The question of the after care of the feeble-minded is most important. Statistics show that a very small percentage of the children leaving our special schools are capable of earning their own living without 


\section{Medical Inspection: Relationship to Home Life of Children.}

supervision. As the recommendations of the Royal Commission do not appear to be likely to become law at an early date, the formation of after-care committees in every city is really an urgent one. Many of these children are quite capalle of undertaking such work as gardening, farm work, cobbling, etc., but unless supervision is exercised they simply recruit the criminal classes.

In conclusion, I think you will agree that medical inspection thoroughly carried out, with the help of the school nurse and the woman inspector, will result in the cleansing of verminous children, and, what is equally important, the cleansing of dirty homes.

It will bring forward the great necessity of increased instruction in such suljects as housewifery and cookery, and by observations, statistical and otherwise, will bring strong arguments to bear on the necessity for social reforms, which will make it possible for all school children to live in houses in which they can be properly washed, properly fed, and obtain sufficient sleep, houses in other words which deserve the title of homes.

Dr. H. Peok (Chesterfeld) said he thought that a 98 percentage of children troubled with pediculi to be extraordinarily large, but supposed it was due to the children being girls and having long hair. He had experience of isolation bospitals, and found that about 80 per cent. of the child patients were so affected. Children in Chesterfield had been found during the medical inspection of school children to be troulsled this way to the extent of 69 per cent., girls more so thin boys. He thought baths should be provided at schools, and the children taught to use them. The parents often did not know how. He had known a row of cottages built by an enterprising builder who put a little scullerywashhouse at the back of each, with a copper, and a bath at the side of that. The result was that in most cases the baths were used to store lumber, in one case as a dust-bin, and the owner had to take them out.

Dr. C. W. HUTr (Warrington) said that the question very often was a sociological one, as had been pointed out by Dr. Scurfield. How could the wife of a working man earning $17 \mathrm{~s}$. a week, possessing a large family and living in a manufacturing town, keep her children clean?

Due regard should be paid to the difficulty of getting the heads clean. In Chorlton Union Hospitals in Manchester he had seen cases in which, despite the most energetic treatment by nurses skilled in the art of removing nits from the hair, they were present as long as three weeks after admission. With school girls, plaiting of the hair was a preventive method to be adrocated; with boys 
attending infants' schools, the mothers should be persuaded at as early a date as possible to have their boys bair cut short.

At the time of the actual inspection the greatest care should be taken that nothing should be done which might prejudice the parents against the inspection. They could refuse at any time to allow the children to be examined, and possibly for this reason it would be better for all communications in reference to verminous conditions to be by letter or by means of the school nurse at the house.

The extension of the school age to 15 , and the tenching of manual work as advocated in the majority Report of the Hoor Law Commission, were excellent suggestions from both the physical and moral points of view, and the sooner they were adopted the better.

Dr. H. Scurrield (Sheffield) said that one of the greatest needs of the present day was for stringent metbods of dealing with neglectful parents. If it were possible to remove the children from such parents, and train them apart, regardless of cost, the question would be solved in one generation. He thougbt that in the schools in the poor districts it was advisable that shower baths should be provided. The medical inspection of school children had shown them what an enormous task lay before them.

Dr. J. Mitcheld Wilson, Rev. J.S. Fowle, Dr. Sadler, Mr. W. Bennetr, Mr. C. R. Mclean, Mrs. Franks, Mr. W. H. Harrison, and Mr. H. Clay also took part in the discussion. 